13 Talukdar R, Inoue H, Reddy DN. Efficacy of peroral endoscopic myotomy (POEM) in the treatment of achalasia: a systematic review and meta-analysis. Surg Endosc 2014, epub ahead of print.

14 Vanuytsel T, Bisschops R, Farré R et al. Botulinum toxin reduces Dysphagia in patients with non-achalasia primary esophageal motility disorders. Clin Gastroenterol Hepatol 2013;11:1115-21.

15 Fox M, Sweis R, Wong T, Anggiansah A. Sildenafil relieves symptoms and normalizes motility in patients with oesophageal spasm: a report of two cases. Neurogastroenterol Motil 2007;19:798-803.

16 Louis H, Covas A, Coppens E, Devière J. Distal esophageal spasm treated by peroral endoscopic myotomy. Am J Gastroenterol 2012;107:1926-7.

17 Rosołowski M, Kierzkiewicz M. Etiology, diagnosis and treatment of infectious esophagitis. Prz Gastroenterol 2013;8:333-7.

18 Jeong ES, Kim MJ, Yoo SH et al. Intramural hematoma of the esophagus after endoscopic pinch biopsy. Clin Endosc 2012;45:417-20.
19 Blencowe NS, Strong S, Hollowood AD. Spontaneous oesophageal rupture. BMJ 2013;346:f3095.

20 Griffin SM, Lamb PJ, Shenfine J et al. Spontaneous rupture of the oesophagus. Br J Surg 2008;95:1115-20.

21 Schweigert M, Beattie R, Solymosi N et al. Endoscopic stent insertion versus primary operative management for spontaneous rupture of the esophagus (Boerhaave syndrome): an international study comparing the outcome. Am Surg 2013;79:634-40.

22 Kobara H, Mori H, Rafiq K et al. Successful endoscopic treatment of Boerhaave syndrome using an over-the-scope clip. Endoscopy 2014;46:E82-3.

Address for correspondence: $\mathrm{Dr}]$ de Caestecker, Leicester General Hospital, Gwendolen Road, Leicester LE5 4PW, UK. Email: john.decaestecker@uhl-tr.nhs.uk

\title{
Acute upper gastrointestinal bleeding
}

\author{
Authors: Matthew Kurien ${ }^{A}$ and Alan J Lobo ${ }^{B}$
}

Acute upper gastrointestinal bleeding (AUGIB) is a frequently encountered medical emergency with an incidence of 84$160 / 100,000$ and associated with mortality of approximately $10 \%$. Guidelines from the National Institute for Care and Care Excellence outline key features in the management of AUGIB. Patients require prompt resuscitation and risk assessment using validated tools. Upper gastrointestinal endoscopy provides accurate diagnosis, aids in estimating prognosis and allows therapeutic intervention. Endoscopy should be undertaken immediately after resuscitation in unstable patients and within 24 hours in all other patients. Interventional radiology may be required for bleeding unresponsive to endoscopic intervention. Drug therapy depends on the cause of bleeding. Intravenous proton pump inhibitors should be used in patients with highrisk ulcers. Terlipressin and broad-spectrum antibiotics should be used following variceal haemorrhage. Hospitals admitting patients with AUGIB need to provide well organised services and ensure access to relevant services for all patients, and particularly to out of hours endoscopy.

Authors: Aacademic clinical lecturer in gastroenterology, Department of Gastroenterology, Royal Hallamshire Hospital, Sheffield, UK, and Academic Unit of Gastroenterology, University of Sheffield, Beech Hill Rd, Sheffield, UK; ${ }^{B}$ consultant gastroenterologist, Sheffield Teaching Hospitals NHS Foundation Trust, Sheffield, UK, and professor of gastroenterology, Academic Unit of Gastroenterology, University of Sheffield, Sheffield, UK

\section{Introduction}

Acute upper gastrointestinal bleeding (UGIB) is a common medical emergency characterised by haematemesis and/or melaena. Massive haemorrhage from the upper GI tract may be associated with brighter rectal bleeding. Haemodynamic instability may also feature, with patients presenting with dizziness, syncope or in hypovolaemic shock. Despite the declining incidence of UGIB, advances in therapeutic endoscopy and increased use of acid suppressing medication, there is a significant in-hospital mortality. ${ }^{1}$ This review provides an overview of UGIB incorporating current standards for its management (Box 1).

\section{Epidemiology}

The incidence of UGIB is between 84 and 160 cases per 100,000 adults in European populations, with incidence highest in men, in lower socioeconomic groups and in the elderly. ${ }^{2}$ Peptic ulcer bleeding is the commonest identified cause, though its incidence is declining - possibly due to the use of proton pump inhibitors (PPIs) and the decreasing prevalence of Helicobacter pylori infection. By contrast, the relative contribution of variceal bleeding appears to be rising in the UK, accounting for $11 \%$ of all UGIB admissions in the 2007 national audit, ${ }^{1}$ compared to only $4 \%$ in $1993 .{ }^{3}$ Mortality in the successive national audits has appeared to fall from $14 \%$ to $10 \% .{ }^{1,3}$

\section{Pre-endoscopy care}

\section{Assessment and risk stratification}

All patients presenting with UGIB require prompt assessment using a validated assessment tool. Early assessment identifies 


\section{Risk assessment}

1. Use the following formal risk assessment scores for all patients with UGIB:

$>$ Blatchford score at first assessment

$>$ full Rockall score after endoscopy.

\section{Timing of endoscopy}

1. Offer endoscopy to unstable patients with severe UGIB immediately after resuscitation.

2. Offer endoscopy within 24 hours of admission to all other patients with UGIB.

3. Units seeing $>330$ cases a year should offer daily endoscopy lists. Units seeing $<330$ cases a year should arrange their service according to local circumstances.

\section{Management of non-variceal bleeding}

1. Do not use adrenaline as monotherapy for the endoscopic treatment of non-variceal UGIB.

2. For the endoscopic treatment of non-variceal UGIB, use one of the following:

$>$ a mechanical method (for example, clips) with or without adrenaline

$>$ thermal coagulation with adrenaline

$>$ fibrin or thrombin with adrenaline.

3. Offer interventional radiology to unstable patients who re-bleed after endoscopic treatment. Refer urgently for surgery if interventional radiology is not promptly available.

\section{Management of variceal bleeding}

1. Offer prophylactic antibiotic therapy at presentation to patients with suspected or confirmed variceal bleeding.

2. Consider TIPS if bleeding from oesophageal varices is not controlled by band ligation.

\section{Control of bleeding and prevention of re-bleeding in patients on NSAIDs, aspirin or clopidogrel}

1. Continue low-dose aspirin for secondary prevention of vascular events in patients with UGIB in whom haemostasis has been achieved.

NICE = National Institute for Health and Care Excellence; NSAIDs = non-steroidal anti-inflammatory drugs; TIPS = transjugular intrahepatic portosystemic shunts; UGIB = upper gastrointestinal bleeding.

\section{Key points}

Acute upper gastrointestinal bleeding (UGIB) is associated with significant mortality $-10 \%$ in the most recent UK national audit.

Patients with suspected UGIB should promptly be assessed and risk stratified using either the Blatchford or full postendoscopy Rockall scores.

All patients with UGIB should be resuscitated prior to endoscopy, aiming to correct fluid losses and restore haemodynamic stability.

High-risk patients should undergo endoscopy urgently following resuscitation, with other UGIB patients undergoing endoscopy within 24 hours.

Organisation of UGIB services within the UK should ensure comprehensive care for all patients presenting with acute UGIB, and adequate training provision.

KEYWORDS: Gastrointestinal bleeding, varices, peptic ulcer disease, endoscopy, mortality, UGIB patients at high risk of death, of further bleeding and those requiring intervention, including surgery. Many predictive tools have been described for risk stratification of people with UGIB, but there is substantial variation in the outcomes assessed and in methodological quality. ${ }^{4}$ The National Institute for Health and Care Excellence advocates use of the Glasgow-Blatchford Score (GBS) at initial assessment (Table 1) and the full Rockall score after endoscopy to risk stratify all UGIB patients (Table 2). ${ }^{2}$ Both scores perform similarly in predicting mortality; however GBS predicts an individual's need for intervention and transfusion more accurately, and may therefore be more appropriate as a guide for early clinical decisions. ${ }^{5}$ Low-risk patients with a GBS of zero can be safely managed without admission. ${ }^{6}$

\section{Resuscitation}

Early, intensive resuscitation significantly reduces mortality in UGIB. ${ }^{7}$ Haemodynamic stability should be restored using intravenous fluids (crystalloids or colloids), alongside blood transfusion, oxygen therapy and correction of coagulopathy where appropriate.

A restrictive blood transfusion strategy, which aims for haemoglobin concentration between $70-90 \mathrm{~g} / \mathrm{L}$, has been associated with improved six-week survival (95 vs 91\%) and reduced rebleeding (10 vs 16\%), compared with a more liberal 
Table 1. Risk stratification score: GlasgowBlatchford Score. ${ }^{2,6}$

\begin{tabular}{|c|c|}
\hline Admission risk factor & Score \\
\hline \multicolumn{2}{|l|}{ Blood urea } \\
\hline $6.5-7.9$ & 2 \\
\hline $8.0-9.9$ & 3 \\
\hline $10.0-25.0$ & 4 \\
\hline$>25.0$ & 6 \\
\hline \multicolumn{2}{|l|}{ Haemoglobin for men (g/L) } \\
\hline $120-129$ & 1 \\
\hline $100-119$ & 3 \\
\hline$<100$ & 6 \\
\hline \multicolumn{2}{|l|}{ Haemoglobin for women ( $\mathrm{g} / \mathrm{L}$ ) } \\
\hline $100-119$ & 1 \\
\hline$<100$ & 6 \\
\hline \multicolumn{2}{|l|}{ Systolic blood pressure (mmHg) } \\
\hline 100-109 & 1 \\
\hline $90-99$ & 2 \\
\hline$<90$ & 3 \\
\hline \multicolumn{2}{|l|}{ Other markers } \\
\hline Pulse $\geq 100 \mathrm{bpm}$ & 1 \\
\hline Presentation with melaena & 1 \\
\hline Presentation with syncope & 2 \\
\hline Hepatic disease & 2 \\
\hline Cardiac failure & 2 \\
\hline
\end{tabular}

strategy with a haemoglobin target of $90-110 \mathrm{~g} / \mathrm{L} .{ }^{8}$ However, the findings were from a single centre where all patients received endoscopy within six hours of admission and many high-risk patients were excluded. Nevertheless, the findings do support previous studies in $\mathrm{UGIB}^{9}$ and evidence from orthopaedic and critical care literature that a restrictive red cell transfusion strategy may be beneficial within the context of acute bleeding.

\section{Early pharmacotherapy}

Pharmacotherapy prior to endoscopy for UGIB may be influenced by the suspected cause. Where there is a high suspicion of variceal bleeding (those with established cirrhosis, signs of liver disease or previous variceal bleeding), intravenous terlipressin reduces mortality and the requirement for additional procedures, and improves initial haemostasis. ${ }^{2}$ These patients are also at increased risk of bacterial infection, with the risk highest in those with advanced liver disease (Child B and C). All patients with suspected variceal bleeding should therefore be considered for broad-spectrum antibiotics, with the agent used determined by local antibiotic resistance patterns. ${ }^{2}$

In the overall cohort of those with UGIB, use of PPIs before endoscopy does not reduce mortality, surgery or rebleeding. ${ }^{10}$ However, stigmata of recent haemorrhage and the need for intervention at index endoscopy may be reduced, suggesting PPIs might have a role when endoscopy is delayed or cannot be undertaken. ${ }^{10}$ Intravenous erythromycin, through its prokinetic action, may optimise mucosal views, reduce the need for a second look endoscopy and reduce blood transfusion requirements; however, it has not been widely recommended. ${ }^{11}$

\section{Endoscopy}

\section{Timing of endoscopy}

Endoscopy following UGIB is well established in clinical practice, giving a diagnosis, prognostic detail and permitting therapeutic intervention. Urgent endoscopy should be undertaken immediately after stabilisation of initially unstable patients, and within 24 hours of admission for all other UGIB patients. ${ }^{2}$ Evidence to support this is difficult to interpret. Although observational studies suggest a benefit of endoscopy within 24 hours following admission in reducing hospital stay and need for surgical intervention, ${ }^{12}$ the inability to demonstrate a clear benefit for early endoscopy may be due to the case mix in randomised controlled trials ${ }^{11}$ or uncontrolled confounding variables in the UK national audit. ${ }^{1,2}$

\section{Endoscopic interventions}

For ulcer bleeding, endoscopic therapy is required if active bleeding is identified or if endoscopic features suggest a high risk of rebleeding. High-risk features include ulcers with an active, spurting vessel, non-bleeding visible vessel, active oozing or adherent clot. ${ }^{12}$ Therapy comprises a combination of adrenaline

\section{Table 2. Risk stratification score: full post-endoscopy Rockall score. ${ }^{2}$}

\section{Risk factor}

\begin{tabular}{lllll}
\cline { 2 - 4 } & $\mathbf{0}$ & $\mathbf{1}$ & $\mathbf{2}$ & $\mathbf{3}$ \\
Age, years & $<60$ & $60-79$ & $>80$ & \\
Shock & No shock & Pulse $>100 \mathrm{bpm}$ & Systolic blood pressure $<100 \mathrm{mmHg}$ & Renal failure, liver failure, metastatic \\
Comorbidity & Nil major & & CHF, IHD, major morbidity & cancer \\
Diagnosis & Mallory-Weiss & All other diagnoses & GI malignancy & Blood, adherent clot, spurting vessel \\
Evidence of bleeding & None & & S
\end{tabular}


Fig 1. (a) Endoscopic appearance of oesophageal varices. (b) Endoscopic appearance of oesophageal varices after endoscopic band ligation.

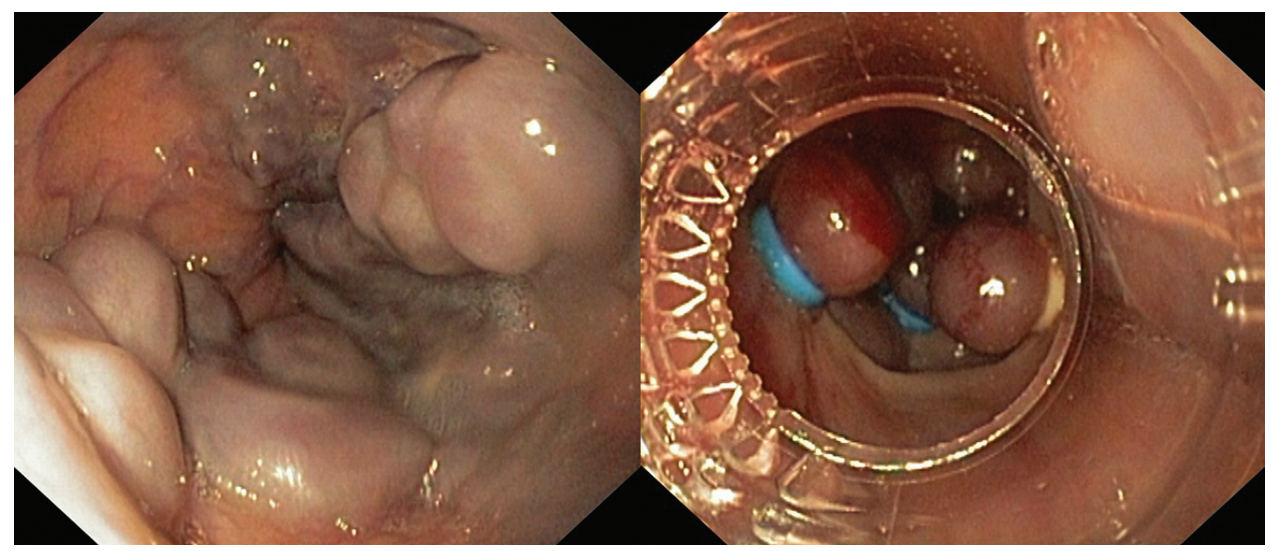

injection to the ulcer with another modality: either mechanical (eg endoscopic clips) or thermal (eg bipolar electrocoagulation, heater probe) to achieve haemostasis. ${ }^{2,12}$ If haemostasis is not achieved at endoscopy for non-variceal UGIB, there should be early consideration of interventional radiology (angiography \pm embolisation) or surgery in unstable patients, depending on local availability and expertise. ${ }^{2}$ If patients rebleed having achieved initial haemostasis at endoscopy, then a further attempt at endoscopic control of bleeding should be made. ${ }^{12}$ Both failed primary haemostasis and rebleeding are associated with increased mortality. ${ }^{2}$ Novel endoscopic haemostatic devices or sprays may be useful adjuncts but more trial data are required to establish their position in clinical practice.

The first-line treatment for oesophageal varices is endoscopic band ligation, and for gastric varices is intravariceal injection of cyanoacrylate glue. Continued bleeding or early rebleeding despite initial endoscopic treatment occurs in 10-20\% of patients and balloon tamponade, as a temporising measure, or a transjugular intrahepatic portosystemic shunt may be required. ${ }^{13}$

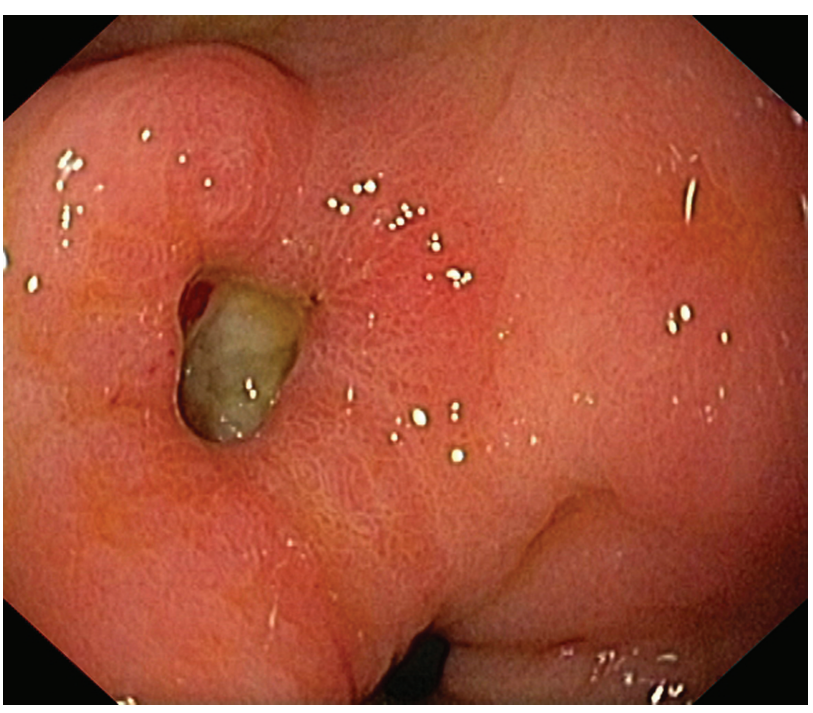

Fig 2. Gastric ulcer close to the pylorus.

\section{Post-endoscopy care}

\section{Pharmacotherapy}

Following haemostasis, all patients with high-risk ulcers should be commenced on intravenous PPI therapy for 72 hours. Following this, twice daily PPI for a further 11 days may be beneficial. ${ }^{14}$ For low-risk ulcers (clean base, flat pigmented spots only), once daily, oral PPI is appropriate. H pylori eradication given alongside acid suppression in those positive for the organism on mucosal biopsy reduces the risk of subsequent rebleeding. ${ }^{12}$

Patients on antiplatelet therapy, anticoagulants and nonsteroidal anti-inflammatory drugs (NSAIDs) prior to UGIB require special attention. Currently, international guidance recommends withholding aspirin until haemostasis is achieved and restarting within 7 days (ideally $1-3$ days) if it is needed for secondary prevention of vascular events. ${ }^{2,12}$ The benefits and risks of continuing clopidogrel in the context of UGIB should be made following consultation with the patient and relevant specialties (cardiology and haematology).

When an NSAID may have caused ulcer bleeding, the NSAID should be withheld during the acute phase and their indication reviewed. ${ }^{2}$ If NSAIDs need to be continued then a cyclooxygenase-2-selective NSAID at the lowest effective dose plus daily PPI is recommended. ${ }^{12}$ There is a paucity of data on the management of anticoagulants (warfarin, rivaroxaban and dabigatran) following UGIB. The drug would usually be withheld with reintroduction dependent upon thrombotic risk and influenced by rebleeding risk. ${ }^{15}$

Following variceal haemorrhage, terlipressin should be continued for 3-5 days after endoscopic treatment and haemostasis. Patients should then be considered for secondary prophylaxis including non-selective $\beta$-blockers and surveillance endoscopy with endoscopic band ligation.

\section{Organisation of UGIB services}

Hospitals need a robust infrastructure to deliver care for patients with UGIB, which includes 24-hour access to endoscopy, interventional radiology and surgical intervention, and to minimise variations in outcome related to out-of-hours admission. ${ }^{16}$ However, such access may not be universally available - with just $52 \%$ of UK hospitals having a formal, consultant-led, out-of-hours endoscopy service in the 2007 
UGIB National Audit. Risk-adjusted mortality was higher in hospitals without such rotas. ${ }^{17}$ Provision may be improving with $67 \%$ of hospitals reporting provision of an out-of-hours rota in 2013 , though just $56 \%$ reported that all patients are offered upper GI endoscopy within 24 hours. ${ }^{18}$ The challenge of improving this access through expansion of the numbers of hospitals providing out of hours endoscopy, alone or as a network, remains a priority. ${ }^{18} \mathrm{~A}$ gastrointestinal haemorrhage unit supports delivery of care and improves outcomes, focusing clinical expertise and allowing rapid recognition of clinical deterioration or rebleeding and co-ordination of each stage of a patient's management. ${ }^{19}$ Sustainability of services for UGIB also requires that those appointed to consultant posts have had adequate training and experience. ${ }^{20}$

\section{Acknowledgements}

The authors would like to thank Dr AD Hopper (consultant gastroenterologist, Sheffield Teaching Hospitals NHS Foundation Trust) for providing the images in Figures 1 and 2.

\section{References}

1 Hearnshaw SA, Logan RF, Lowe D et al. Acute upper gastrointestinal bleeding in the UK: patient characteristics, diagnoses and outcomes in the 2007 UK audit. Gut 2011;60:1327-35.

2 National Institute for Health and Care Excellence. Acute upper gastrointestinal bleeding: management (CG141). London: NICE, 2012.

3 Rockall TA, Logan RF, Devlin HB et al. Incidence of and mortality from acute upper gastrointestinal haemorrhage in the United Kingdom. Steering Committee and members of the National Audit of Acute Upper Gastrointestinal Haemorrhage. BMJ 1995;311:222-6.

4 de Groot NL, Bosman JH, Siersema PD et al. Prediction scores in gastrointestinal bleeding: a systematic review and quantitative appraisal. Endoscopy 2012;44:731-9.

5 Stanley AJ, Dalton HR, Blatchford O et al. Multicentre comparison of the Glasgow Blatchford and Rockall Scores in the prediction of clinical end-points after upper gastrointestinal haemorrhage. Aliment Pharmacol Ther 2011;34:470-5.

6 Stanley AJ, Ashley D, Dalton HR et al. Outpatient management of patients with low-risk upper-gastrointestinal haemorrhage: multicentre validation and prospective evaluation. Lancet 2009;373:42-7.

7 Baradarian R, Ramdhaney S, Chapalamadugu R et al. Early intensive resuscitation of patients with upper gastrointestinal bleeding decreases mortality. Am J Gastroenterol 2004;99:619-22.
8 Villanueva C, Colomo A, Bosch A et al. Transfusion strategies for acute upper gastrointestinal bleeding. N Engl J Med 2013;368:11-21.

9 Jairath V, Hearnshaw S, Brunskill SJ et al. Red cell transfusion for the management of upper gastrointestinal haemorrhage. Cochrane Database Syst Rev 2010:CD006613.

10 Sreedharan A, Martin J, Leontiadis GI et al. Proton pump inhibitor treatment initiated prior to endoscopic diagnosis in upper gastrointestinal bleeding. Cochrane Database Syst Rev 2010:CD005415.

11 Barkun AN, Bardou M, Kuipers EJ et al. International consensus recommendations on the management of patients with nonvariceal upper gastrointestinal bleeding. Ann Intern Med 2010;152:101-13.

12 Laine L, Jensen DM. Management of patients with ulcer bleeding. Am J Gastroenterol 2012;107:345-60.

13 Garcia-Tsao G, Sanyal AJ, Grace ND et al. Prevention and management of gastroesophageal varices and variceal hemorrhage in cirrhosis. Am J Gastroenterol 2007;102:2086-102.

14 Cheng HC, Wu CT, Chang WL et al. Double oral esomeprazole after a 3-day intravenous esomeprazole infusion reduces recurrent peptic ulcer bleeding in high-risk patients: a randomised controlled study. Gut 2014;63:1864-72.

15 Woodhouse C, Evans G, Muller AF. The new oral anticoagulants: practical management for patients attending for endoscopic procedures. Frontline Gastroenterol 2013;4:213-8.

16 de Groot NL, Bosman JH, Siersema PD et al. Admission time is associated with outcome of upper gastrointestinal bleeding: results of a multicentre prospective cohort study. Aliment Pharmacol Ther 2012;36:477-84.

17 Hearnshaw SA, Logan RF, Lowe D et al. Use of endoscopy for management of acute upper gastrointestinal bleeding in the UK: results of a nationwide audit. Gut 2010;59:1022-9.

18 British Society of Gastroenterology. Acute upper gastrointestinal bleeding an overview of out of hours service provision and equity of access. London: NHS Improving Quality, 2014.

19 Sanders DS, Perry MJ, Jones SG et al. Effectiveness of an uppergastrointestinal haemorrhage unit: a prospective analysis of 900 consecutive cases using the Rockall score as a method of risk standardisation. Eur J Gastroenterol Hepatol 2004;16:487-94.

20 Penny HA, Kurien M, Wong E et al. Changing trends in the UK management of upper GI bleeding: is there evidence of reduced UK training experience? Frontline Gastroenterol 2015;doi:10.1136/ flgastro-2014-100537.

Address for correspondence: Prof A Lobo, Gastroenterology Unit, P Floor, Royal Hallamshire Hospital, Glossop Road, Sheffield S10 2JF, UK. Email: alan.lobo@sth.nhs.uk 